Laboratory simulations of supernova shockwaves: Formation of a second shock ahead of a radiative shock

J. F. Hansen, M. J. Edwards, D. Froula, G.

Gregori, A. Edens, T. Ditmire

February 3, 2005

Magnetic Fields in the Universe Angra dos Reis, Brazil

November 28, 2004 through December 3, 2004 
This document was prepared as an account of work sponsored by an agency of the United States Government. Neither the United States Government nor the University of California nor any of their employees, makes any warranty, express or implied, or assumes any legal liability or responsibility for the accuracy, completeness, or usefulness of any information, apparatus, product, or process disclosed, or represents that its use would not infringe privately owned rights. Reference herein to any specific commercial product, process, or service by trade name, trademark, manufacturer, or otherwise, does not necessarily constitute or imply its endorsement, recommendation, or favoring by the United States Government or the University of California. The views and opinions of authors expressed herein do not necessarily state or reflect those of the United States Government or the University of California, and shall not be used for advertising or product endorsement purposes. 


\title{
Laboratory simulations of supernova shockwaves: Formation of a second shock ahead of a radiative shock
}

\author{
J. F. Hansen*, M. J. Edwards*, D. Froula*, G. Gregori*, A. Edens ${ }^{\dagger}$ and T. \\ Ditmire $^{\dagger}$ \\ * Lawrence Livermore National Laboratory, Livermore CA 94550, USA \\ ${ }^{\dagger}$ University of Texas at Austin, Austin TX 78712, USA
}

\begin{abstract}
Supernovae launch spherical shocks into the circumstellar medium (CSM). These shocks may interact with both the intergalactic magnetic field (IGM) and local mass accumulations (possibly with their own local magnetic fields). The latter interaction may trigger star formation. The shocks have high Mach numbers and may be radiative. We have created similar shocks in the laboratory by focusing laser pulses onto the tip of a solid pin surrounded by ambient gas; ablated material from the pin rapidly expands and launches a shock through the surrounding gas. The shock may then be allowed to interact with (a) mass accumulations, (b) magnetic fields, or (c) allowed to expand freely. We will present examples of each type of experiment, but mainly discuss a new phenomena observed first in (c); at the edge of the radiatively heated gas ahead of the shock, a second shock forms. The two expanding shocks are simultaneously visible for a time, until the original shock stalls from running into the heated gas. The second shock remains visible and continues to expand. A minimum condition for the formation of the second shock is that the original shock is super-critical, i.e., the temperature distribution ahead of the original shock has an inflexion point. In a non-radiative control experiment the second shock does not form.
\end{abstract}

Keywords: shock, radiative, super critical, interstellar matter, xenon, Taylor, Sedov, Barenblatt, Mach

PACS: $52.72 .+\mathrm{v}, 52.35 . \mathrm{Tc}, 97.60 . \mathrm{Bw}, 98.38 .-\mathrm{j}$

\section{INTRODUCTION}

Interstellar space consists of a tenuous plasma capable of propagating shocks over great distances. Shocks originate in supernova (SN) explosions $[1,2,3,4,5]$ and other astrophysical phenomena (e.g., T Tauri stars and stellar winds) and are important to understand as they mix up circumstellar matter and thus affect mass-loading, stellar formation $[6,7,8]$ and the history of the Milky Way and other galaxies. The shocks have high Mach numbers, can be strongly radiative [9], and can interact with magnetic fields in addition to their interaction with circumstellar material. Until recently, high Mach number, radiative shocks have not been easily attainable in laboratories, but using high-power lasers, shocks with these properties can now be created and studied experimentally $[10,11,4,12,13,14,15,16]$. Examples of two different experiments are given in Fig. 1.

A SN shock expanding through interstellar space sweeps up interstellar material, most of which ends up in a shell just behind the shock. Once more mass has been swept up by the shock than what was initially present, the shock could be regarded as without 
(a)

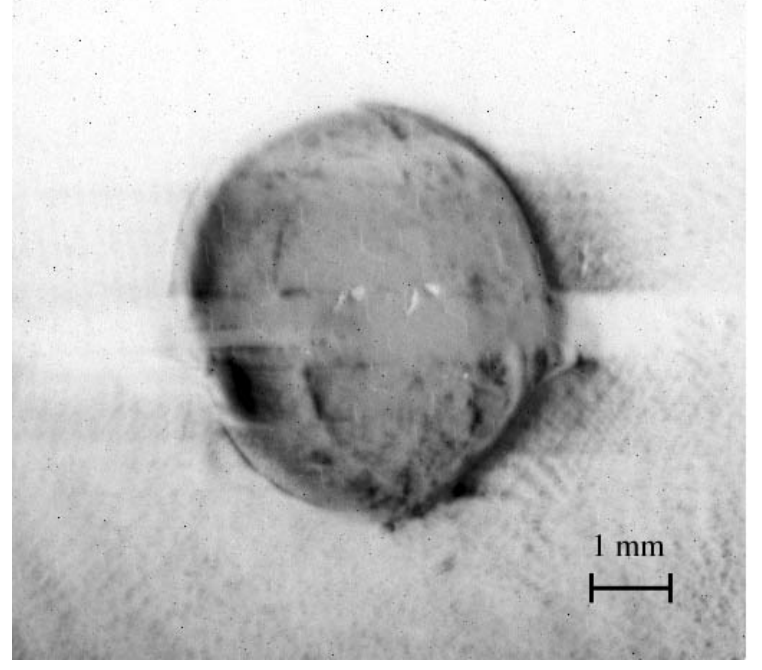

(b)

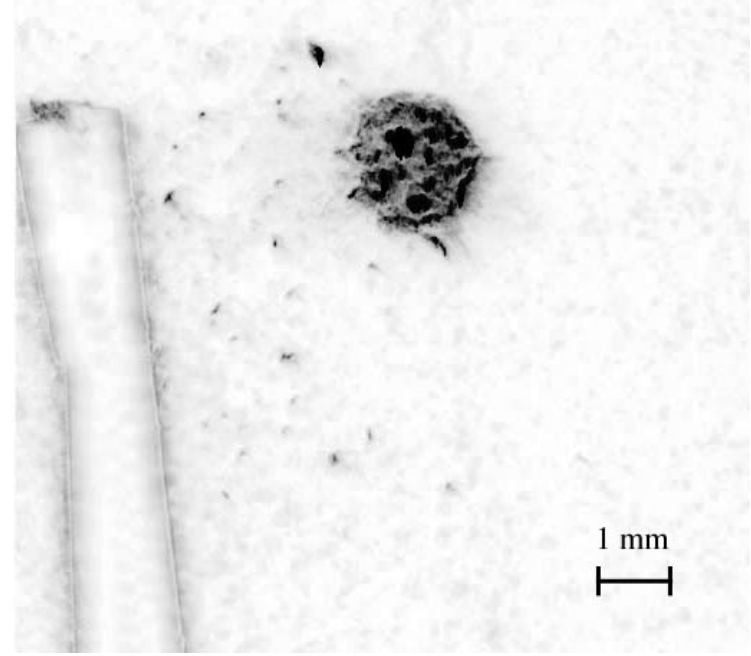

FIGURE 1. Example of two astrophysics shock experiments. To the left (a): a shock in nitrogen $(p=1.39 \mathrm{kPa})$ expanding in a magnetic field $(B=135 \mathrm{mT})$. A point deposit of energy $(E=58.6 \mathrm{~J})$ would normally result in a spherical shock, but the magnetic field, vertical in the image, retards shock expansion resulting in an oval shock. To the right (b): A shock expanding in nitrogen $(p=5.35 \mathrm{kPa})$ has passed a piece of aerogel $\left(\rho=50 \mathrm{mg} / \mathrm{cm}^{3}\right)$ at Mach 3.0. The aerogel (object to the right) is originally held by a metal ring (diffuse object to the left). The image shows the evolution of the aerogel $100 \mu$ s after the interaction.

characteristic length or time scales, and so one would expect the well-known self-similar motion of a Taylor-Sedov blast wave, $r_{s} \propto t^{2 / 5}$, where $r_{s}$ is shock radius and $t$ is time $[17,18,19]$. If radiation removes energy from the shock in an optically thin environment - and SN shocks can be strongly radiative - analytical and numerical studies predict a slower shock expansion, such as $r_{s} \propto t^{2 / 7}$ ("pressure driven snow-plow") and $r_{s} \propto t^{1 / 4}$ ("momentum driven snow-plow"; the shock is simply coasting) [20,9]. Furthermore, a radiative shock is expected to "stall" (vanish) sooner than a non-radiative shock, as its energy is lost.

We have conducted experiments at the Janus laser at the Lawrence Livermore National Laboratory in California, comparing the shock expansion for non-radiative and radiative shocks, and we report here on our findings, including a new phenomena that has not previously been observed.

\section{EXPERIMENTAL SET-UP}

We create spherically expanding blast waves in the following fashion: a high-power infrared pulsed laser (1064 $\mathrm{nm}$ wavelength) is focused onto the tip of a solid (stainless steel) pin surrounded by an ambient gas typically at a pressure of $\sim 1 \mathrm{kPa}$. The laser pulse ( $5 \mathrm{~ns}$ duration and energy ranging from $1 \mathrm{~J}$ to $150 \mathrm{~J}$ ) ablates the pin and rapid expansion of ablated material shocks the ambient gas. The initial shock travels radially outward from the pin, collecting ambient gas in a shell immediately behind the shock front. The 
blast wave velocity drops as more and more of the ambient gas is accumulated and set in motion by the passing shock.

\section{DIAGNOSTICS}

We obtain image and spectrometer data of the shocks to deduce blast wave radius as a function of time and temperature profiles across the shock. To image a blast wave on spatial scales up to $\sim 5 \mathrm{~cm}$, we use two lenses in a telescope configuration and a gated, single-frame, high-speed CCD camera ( 2 ns gate), along with a low energy, green laser pulse ( $\lambda=532 \mathrm{~nm}$ wavelength) as a backlighter. Blast wave radius as a function of time is obtained from schlieren images. A spectrometer is used to infer electron temperatures ahead of and behind the blast wave.

\section{RESULTS WITH NITROGEN AS THE AMBIENT GAS}

Schlieren images were obtained from $5 \mathrm{~ns}$ up to $35 \mu \mathrm{s}$ after the initial laser pulse. Examples of images using nitrogen as the ambient gas can be seen in Fig. 2. In each image, the laser is incident from the left. The pin is clearly visible, as is the expanding blast wave. After an initial, brief, non-self similar phase, the shock expansion settles

into the Taylor-Sedov relationship for a blast wave, $r_{s} \propto\left(\frac{E}{\rho_{0}}\right)^{\frac{1}{5}} t^{\frac{2}{5}}$, where $r_{s}$ is the shock radius, $t$ is time, $E$ is the ablative laser energy, and $\rho_{0}$ is the density of the ambient gas.

Emission spectroscopy data in the near ultraviolet range was readily obtained at and behind the blast wave in nitrogen, but no readings discernible from noise were possible ahead of the shock. Figure 3 shows spectra taken at a time $t=150 \mathrm{~ns}$ after the initial laser pulse $(E=10 \mathrm{~J})$, with electron temperatures in the range $4 \mathrm{eV}$ to $7 \mathrm{eV}$ at and behind the shock.

\section{RESULTS WITH XENON AS THE AMBIENT GAS}

With its higher atomic mass number, xenon radiates more strongly than nitrogen. Therefore, we expected to see a slower shock expansion in xenon, and also to observe the shock stalling. Examples of images obtained with xenon as the ambient gas are shown Fig. 4.

There are several notable differences compared to the images of shocks in nitrogen, all pointing to the radiative nature of the shocks in xenon: (a) plasma emission from preheated gas, that is gas heated by the radiation from the shock, is clearly visible as a glow surrounding the shock at early times $(t \lesssim 400 \mathrm{~ns})$. Spectroscopy data (see Fig. 3 ) confirm that the temperature immediately ahead of the shock is roughly the same as behind the shock. A few millimeters in front of the shock the temperature then drops sharply (to noise levels). (For comparison to the nitrogen case, at $t=150 \mathrm{~ns}$ and a laser energy of $E=10 \mathrm{~J}$ the temperatures in xenon ranged from $2 \mathrm{eV}$ to $5 \mathrm{eV}$.) (b) As expected, the shock rapidly becomes diffuse and stalls, as seen in images from $t \approx 1 \mu \mathrm{s}$ to $4 \mu \mathrm{s}$. (c) Another shock forms ahead of the initial shock. This second shock is a new phenomena that has 


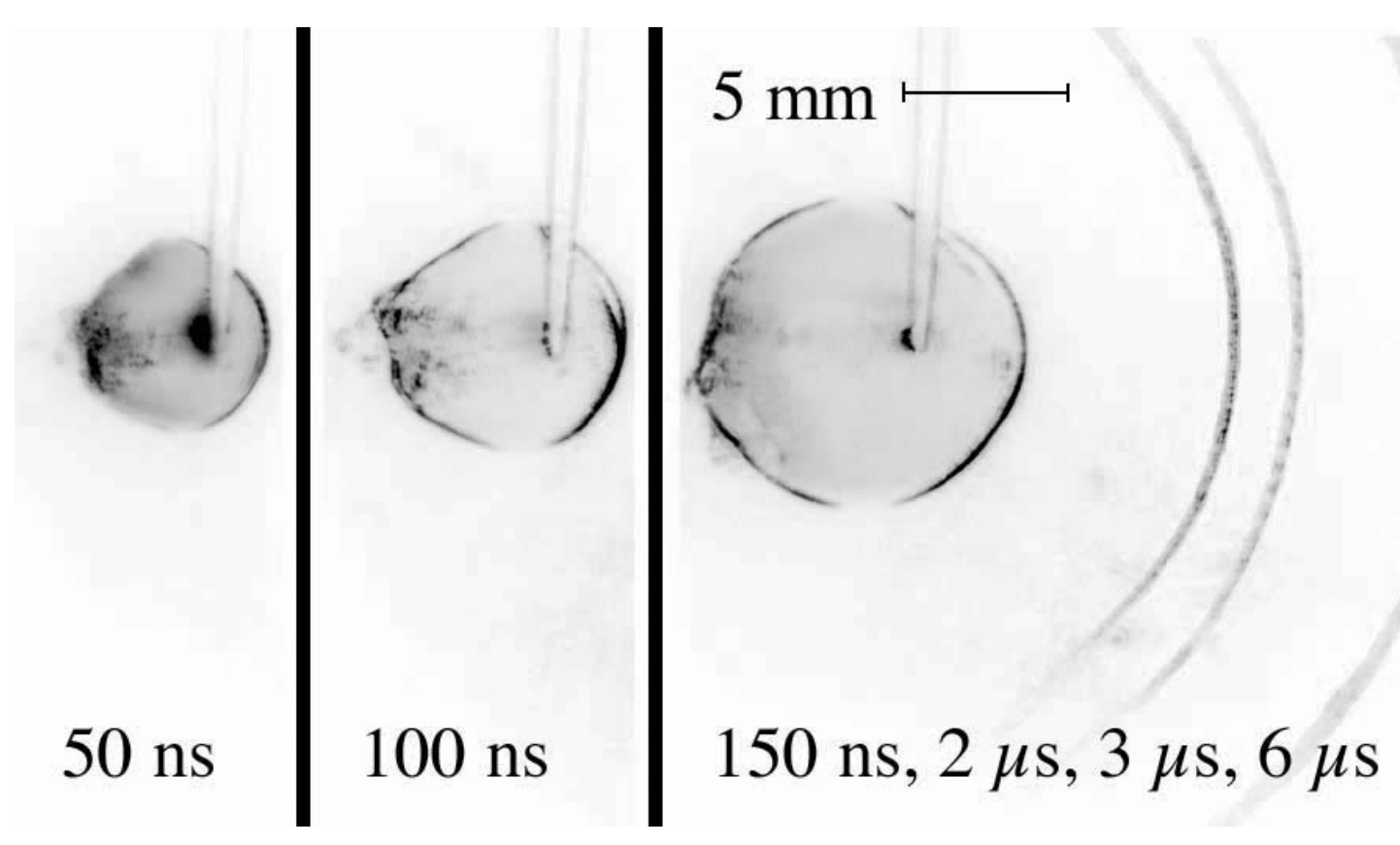

FIGURE 2. Blast wave expansion through nitrogen gas $(1.3 \mathrm{kPa})$ at times $t=150 \mathrm{~ns}$ to $6 \mu \mathrm{s}$ after an ablative laser pulse (energy $E=10 \mathrm{~J}$, duration $5 \mathrm{~ns}$ ) is focused on a solid pin (visible in images, pointed down). The laser pulse was incident from the left. The shock grows as a Taylor-Sedov blast wave. The image to the right ( $t=150 \mathrm{~ns}$ to $6 \mu \mathrm{s}$ ) is a composite of four images (with overlapping pin locations).

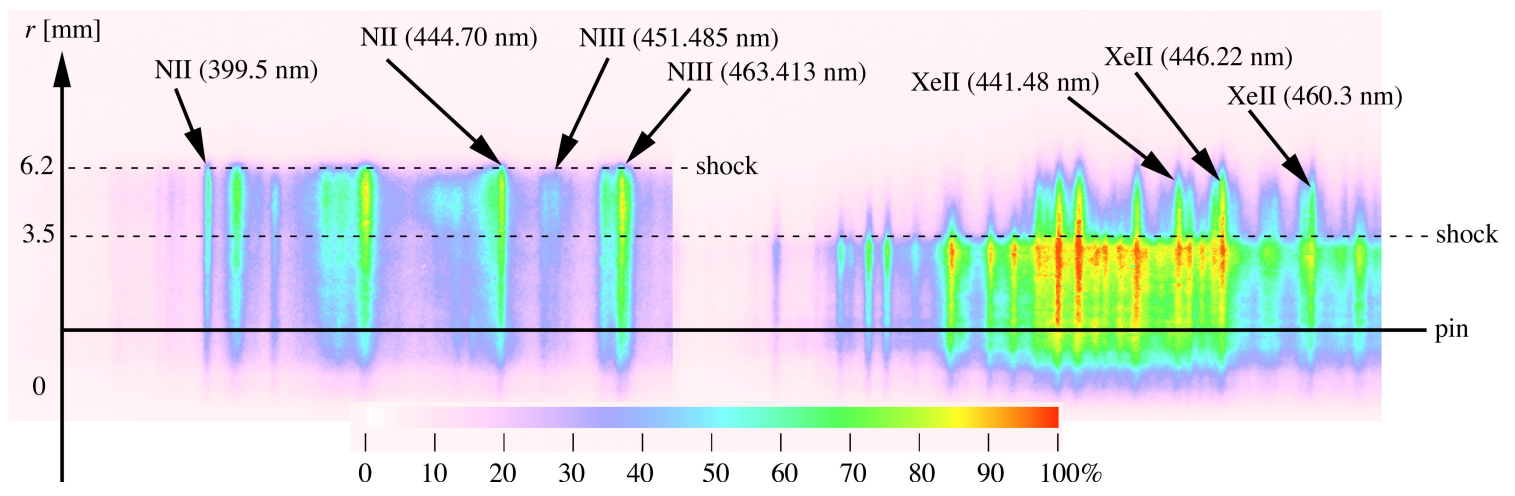

FIGURE 3. Spectra for nitrogen (left) and xenon (right) at $t=150 \mathrm{~ns}$. The location of the pin is marked with a solid line. The shock locations, as obtained from schlieren images, are indicated by dashed lines at radii $r=6.2 \mathrm{~mm}$ for nitrogen and $r=3.5 \mathrm{~mm}$ for xenon.

not previously been discussed in literature. It is not surrounded by a glow of pre-heated gas, and it continues to propagate long after the initial shock has vanished.

Measurements of shock radii versus time is reproduced in Fig. 5.

Despite its radiative nature, the initial shock in xenon appears to follow the TaylorSedov relationship, $r_{s} \propto t^{p}$ with $p=\frac{2}{5}$. This is because most of the radiative losses occurs very early in time (at $t \lesssim 100 \mathrm{~ns}$ ). The second shock is not radiative and follows Taylor-Sedov $p=\frac{2}{5}$. 


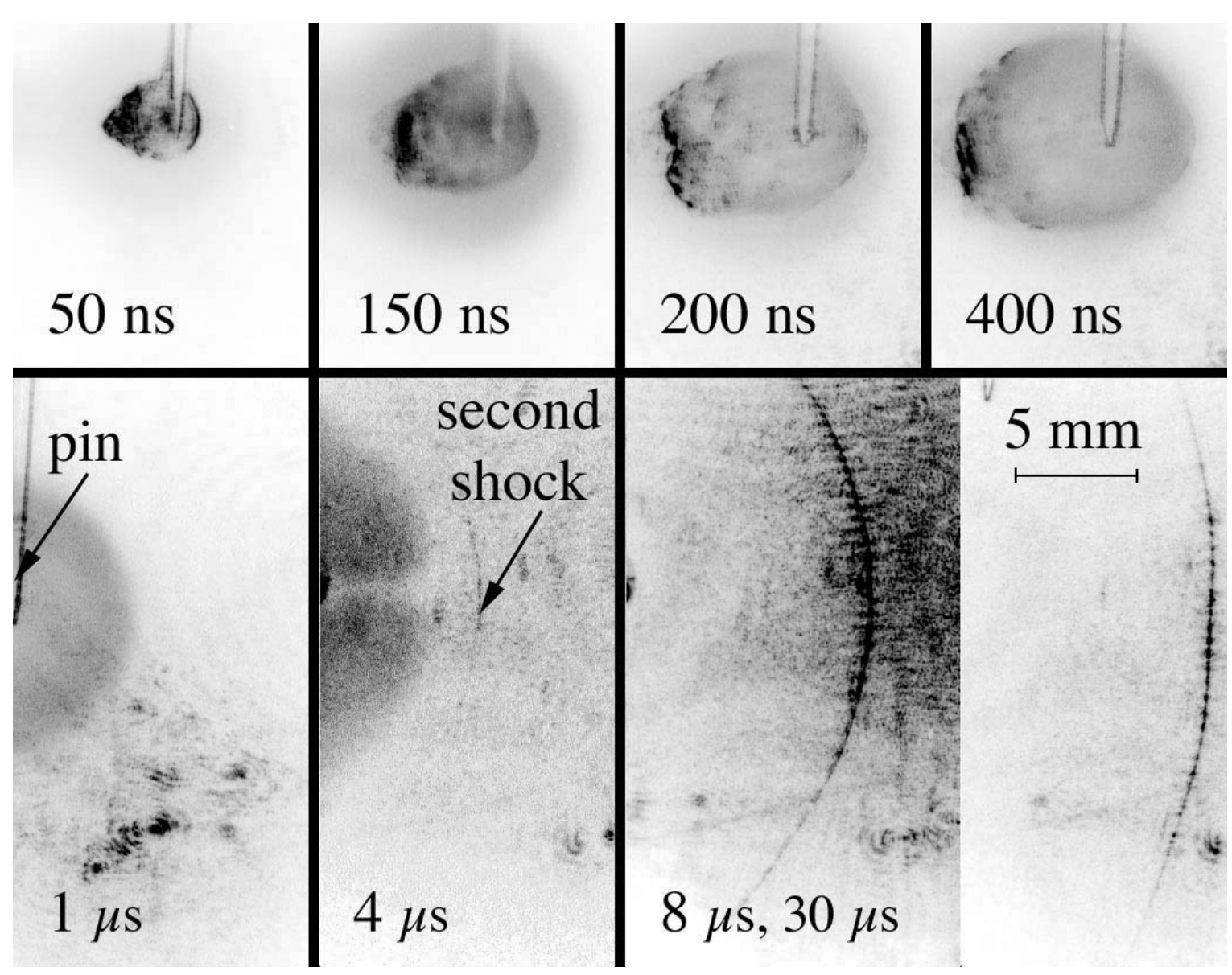

FIGURE 4. Blast wave expansion through ambient Xe gas $(1.3 \mathrm{kPa})$ at times $t=50 \mathrm{~ns}$ to $30 \mu \mathrm{s}$ after an ablative laser pulse (energy $E=10 \mathrm{~J}$, duration $5 \mathrm{~ns}$ ) is focused on a solid pin (visible in images, pointed down; pin location in bottom row of images is at the left edge of each image). The laser pulse was incident from the left. The initial shock is strongly radiative (super-critical) and preheats the ambient gas. At $t=150 \mathrm{~ns}$ both the initial shock and the preheated gas ahead of it are clearly visible. At $t \approx 1 \mu \mathrm{s}$ the initial shock begins to stall, and the shock front is no longer sharp. At $t \approx 4 \mu \mathrm{s}$ a second shock pops out (located at tip of arrow), ahead of the initial shock. The initial shock stalls, while the second shock expands like a Taylor-Sedov blast wave. The final image $(t=8 \mu \mathrm{s}, 30 \mu \mathrm{s})$ is a composite of two images (with overlapping pin locations).

\section{DISCUSSION}

In xenon, the creation of a second shock ahead of the initial shock is a direct consequence of the initial shock being radiative. Figure 6, (computed by the LASNEX code [21] in 1D) shows a plot of compression versus radius in which both shocks are clearly identifiable. The initial shock weakens over time; the second shock grows stronger. In the plot of electron temperature versus radius, the temperature distribution is that of a supercritical shock, with a radiative heat wave moving toward larger radii. The high temperature ahead of the initial shock makes the shock Mach number quite low, resulting in a rapid weakening and shock stall, as can be seen in the compression plot. A vertical comparison 


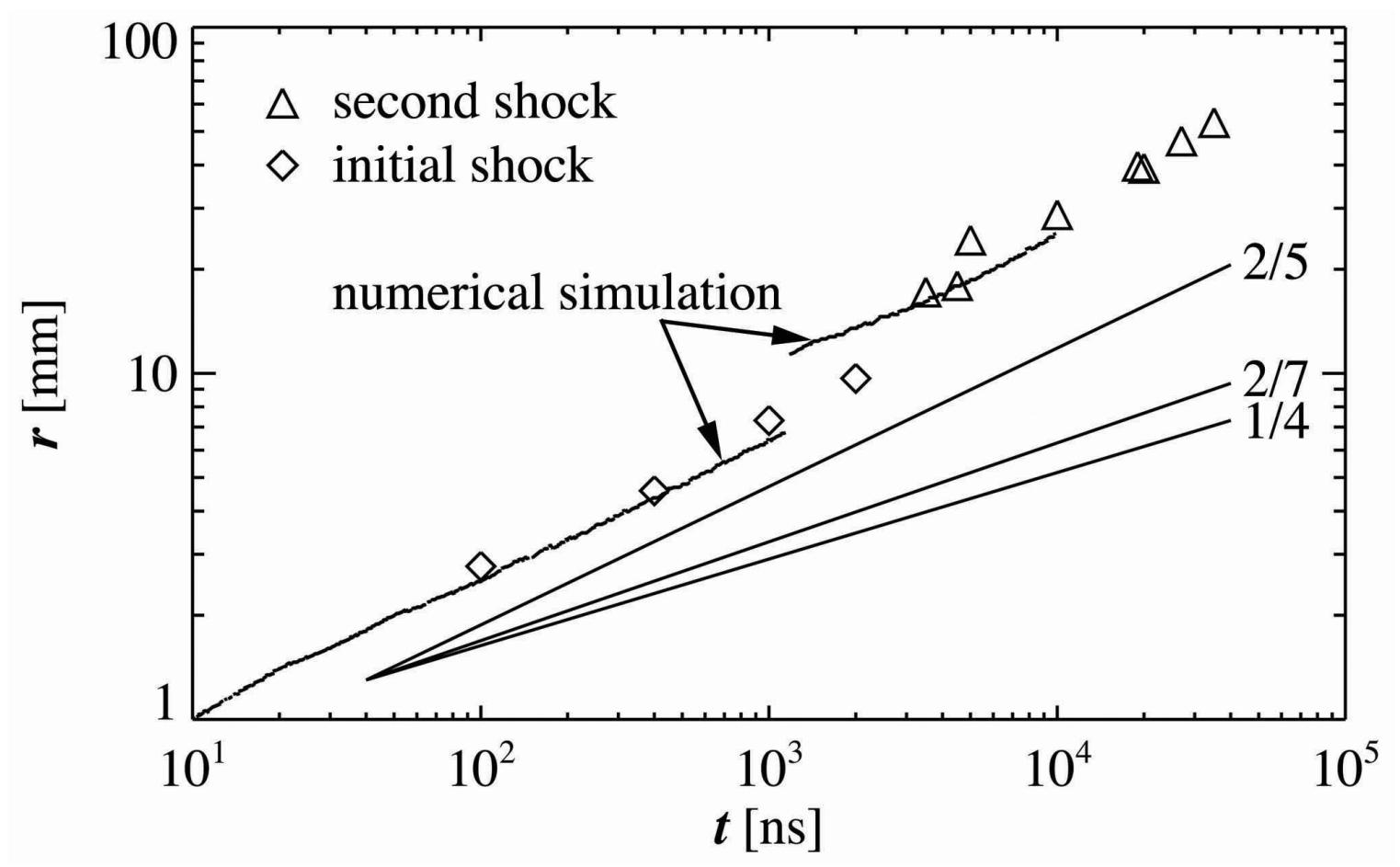

FIGURE 5. Plot of shock radius as a function of time as measured in the experiment, compared to a numerical simulation and to analytical estimates of shock propagation. The 1D numerical simulation by the LASNEX code shows the largest radius at which the compression $\eta>1.25$. The analytical estimates are represented by the three (displaced) lines with slopes $p=\frac{2}{5}, \frac{2}{7}$, and $\frac{1}{4}$.

of the two plots in Fig. 6 shows that the second shock is born at the sharp front of the radiative heat wave. This is confirmed in a second LASNEX calculation where we set up a temperature distribution identical to that in Fig. 6, but excluded the initial shock; with time a shock essentially identical to the second shock in Fig. 6 forms. A third LASNEX calculation with a piston instead of the laser as the initial energy source develops the same temperature distribution and the same second shock, confirming details of initial conditions are not important.

Mathematically, we can make an estimate for where the second shock forms by considering equations for conservation of mass $\rho_{1} u_{1}=\rho_{2} u_{2}$ and momentum $p_{1}+\rho_{1} u_{1}^{2}=$ $p_{2}+\rho_{2} u_{2}^{2}$ in the lab frame of the radiative heat wave front, where subscript 1 denotes the region ahead of the front, and subscript 2 denotes the region behind it. Combining the equations assuming $p_{1} \approx 0$ and $p_{2}=\rho_{2} c_{2}^{2}$, where $c_{2}$ is the sound speed, we find that a real solution of the compression $\eta \equiv \rho_{2} / \rho_{1}$ requires the mixed Mach number $M=u_{1} / c_{2} \geq 2$, so we would expect that the second shock should form at the radiative heat wave front when the velocity of the front drops below Mach 2 (analogous to when a blast wave forms ahead of a fireball $[19,22])$.

To make a numerical comparison to the experiment, we thus need to know the velocity of the radiative heat wave front and the sound speed (or temperature) immediately behind the front. Assuming that we can write the radiative conductivity of the ambient gas as $\chi=\chi_{0} \rho^{a} T^{b}$, then the temperature $T(r, t)$ and the location $r_{h}(t)$ of the radiative heat 


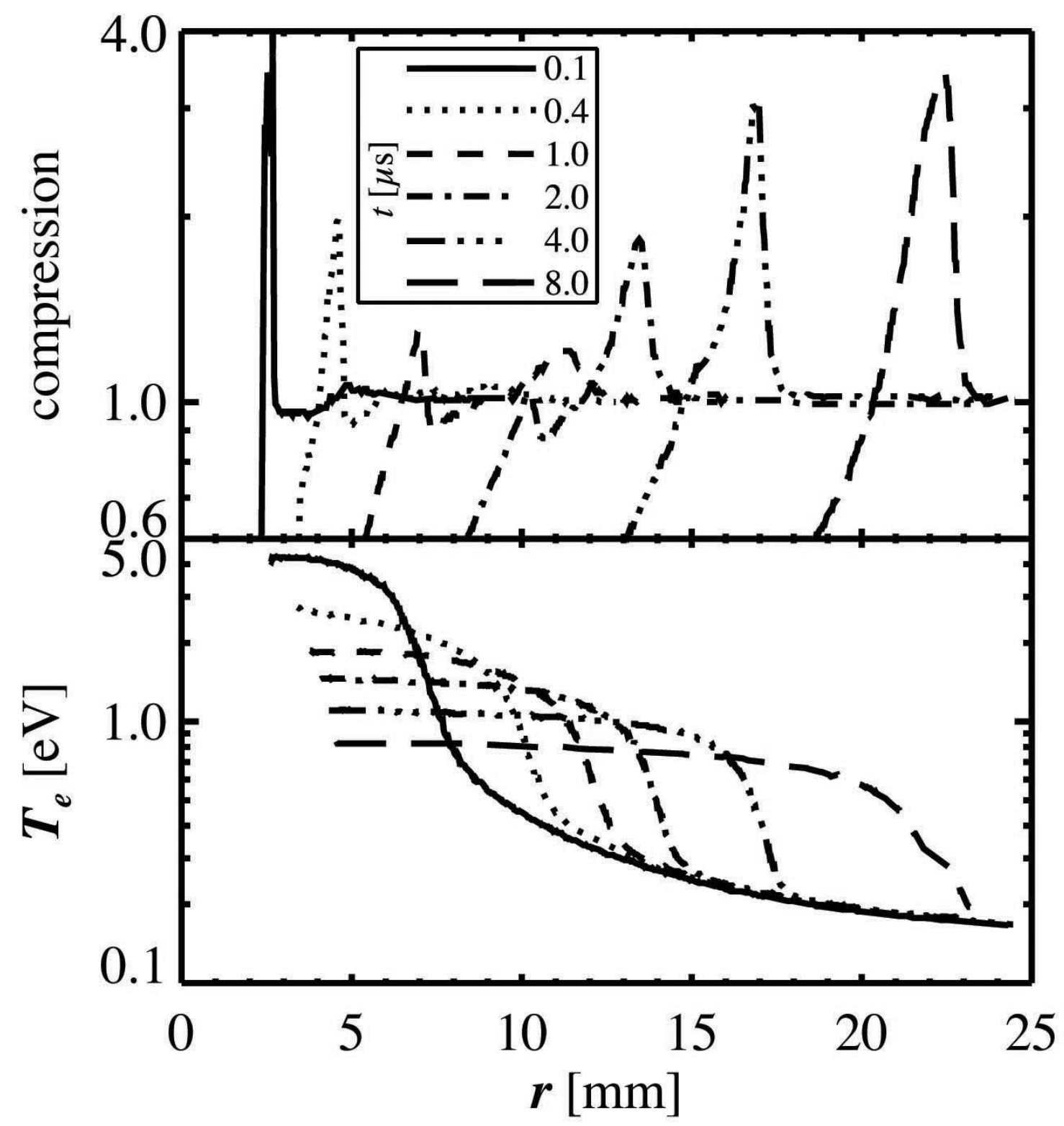

FIGURE 6. Numerical simulation showing compression $\eta$ (upper graph) and electron temperature (lower graph) as a function of radius for six different times. At $t=100$ ns the initial shock has $\eta \approx 6$. Note the initial shock dissipating and the second shock being born at the radiative heat wave front.

wave front is given by Barenblatt's solution [23, 24]. Using values for our experiment in xenon, $E=10 \mathrm{~J}, \rho_{0}=78 \mathrm{~g} / \mathrm{m}^{3}, \gamma=1.2$, and $\chi=10^{-44} \rho^{-2.2} T^{-10}$ in SI-units, we find that the Mach number at the heat front $M\left(r_{h}, t\right)=\frac{d r_{h}(t)}{d t} \sqrt{\frac{m_{0}}{R T\left(r_{h}, t\right)}}$ drops to Mach 2 when $r_{h} \approx 10 \mathrm{~mm}$, consistent with what was actually observed $(\approx 12 \mathrm{~mm})$. 


\section{CONCLUSION}

We have conducted experiments with blast waves traveling through ambient gas, intended to simulate supernova-like shocks. In nitrogen, radiative effects are minimal and the shock expands as a Taylor-Sedov blast wave. In xenon, the shock is strongly radiative, heating the ambient gas ahead of the (supercritical) shock. The loss of energy through radiation and a comparatively low Mach number cause the shock to stall much sooner than the shock in nitrogen. We also report on the first experimental observation of a second shock forming ahead of the initial shock. We show by numerical simulation that the second shock is created at and from the temperature gradient at the front of the slowing radiative heat wave ahead of the initial shock. The second shock is formed at a location that agrees reasonably well with an analytic estimate.

\section{ACKNOWLEDGMENTS}

We thank Dwight Price and the staff at the Janus facility for their valuable assitance. This work was performed under the auspices of the U. S. Department of Energy by the University of California, Lawrence Livermore National Laboratory under Contract No. W-7405-Eng-48.

\section{REFERENCES}

1. E. Müller, B. Fryxell, and D. Arnett, Astron. Astrophys., 251, 505 (1991).

2. J. I. Reed, J. J. Hester, A. C. Fabian, and P. F. Winkler, Astrophys. J., 440, 706 (1995).

3. G. Sonneborn, C. S. J. Pun, R. A. Kimble, T. R. Gull, P. Lundqvist, R. McCray, P. Plait, A. Boggess, C. W. Bowers, A. C. Danks, J. Grady, S. R. Heap, S. Kraemer, D. Lindler, J. Loiacono, S. P. Maran, H. W. Moos, and B. E. Woodgate, Astrophys. J. Lett., 492, L139 (1998).

4. B. A. Remington, D. Arnett, R. P. Drake, and H. Takabe, Science, 284, 1488 (1999).

5. N. Bartel, M. F. Bietenholz, M. P. Rupen, A. J. Beasley, D. A. Graham, V. I. Altunin, T. Venturi, G. Umana, W. H. Cannon, and J. E. Conway, Science, 287, 112 (2000).

6. C. F. McKee, and B. T. Draine, Science, 252, 397 (1991).

7. D. A. Allen, and M. G. Burton, Nature, 363, 54 (1993).

8. R. I. Klein, and D. T. Woods, Astrophys. J., 497, 777 (1998).

9. J. M. Blondin, E. B. Wright, K. J. Borkowski, and S. P. Reynolds, Astrophys. J., 500, 342 (1998).

10. J. C. Bozier, G. Thiell, J. P. LeBreton, S. Azra, M. Decroisette, and D. Schirmann, Phys. Rev. Lett., 57, 1304-1307 (1986).

11. J. Grun, J. Stamper, C. Manka, J. Resnick, R. Burris, J. Crawford, and B. H. Ripin, Phys. Rev. Lett., 66, 2738 (1991).

12. D. Ryutov, R. P. Drake, J. Kane, E. Liang, B. A. Remington, and W. M. Wood-Vasey, Astrophys. J., 518, 821-832 (1999).

13. K. Shigemori, T. Ditmire, B. A. Remington, V. Yanovksy, D. Ryutov, K. G. Estabrook, M. J. Edwards, A. J. MacKinnon, A. M. Rubenchik, K. A. Keilty, and E. Liang, Astrophys. J., 533, 159 (2000).

14. H. F. Robey, J. O. Kane, B. A. Remington, R. P. Drake, O. A. Hurricane, H. Louis, R. J. Wallace, J. Knauer, P. Keiter, D. Arnett, and D. D. Ryutov, Phys. of Plasmas, 8, 2446 (2001).

15. X. Fleury, S. Bouquet, C. Stehle, M. Koenig, D. Batani, A. Benuzzi-Mounaix, J.-P. Chieze, N. Grandjouan, J. Grenier, T. Hall, E. Henry, J.-P. Lafon, S. Leygnac, V. Malka, B. Marchet, H. Merdji, C. Michaut, and F. Thais, Laser and Particle Beams, 20, 263-268 (2002).

16. P. A. Keiter, R. P. Drake, T. S. Perry, H. F. Robey, B. A. Remington, C. A. Iglesias, R. J. Wallace, and J. Knauer, Phys. Rev. Lett., 89, 165003-1-165003-4 (2002).

17. G. I. Taylor, Proc. R. Soc. London A, 201, 159 (1950). 
18. L. I. Sedov, Similarity and Dimensional Methods in Mechanics, Academic, New York, 1959.

19. Y. B. Zeldovich, and Y. P. Raizer, Physics of Shock Waves and High-Temperature Hydrodynamic Phenomena, Academic, New York, 1966.

20. C. F. McKee, and J. P. Ostriker, Astrophys. J., 218, 148 (1977).

21. G. B. Zimmerman, and W. L. Kruer, Comments Plasma Phys. Controlled Fusion, 2, 51 (1975).

22. D. Mihalas, and B. W. Mihalas, Foundations of Radiation Hydrodynamics, Oxford University Press, Oxford, 1984.

23. G. I. Barenblatt, Similarity, Self-Similarity and Intermediate Asymptotics, Consultants Bureau, New York, 1979.

24. P. Reinicke, and J. Meyer-ter-Vehn, Phys. Fluids A, 3, 1807 (1991). 